\title{
IncRNA UASR1 is overexpressed in oral squamous cell carcinoma and regulates cancer cell proliferation by regulating miR-375/JAK2 axis
}

\author{
YUGANG SUN $^{1 *}$, LIANG CHEN ${ }^{2 *}$ and LINA PAN ${ }^{2}$ \\ Departments of ${ }^{1}$ Oral and Maxillofacial Surgery and ${ }^{2}$ Endodontics, Shungeng Branch, \\ Jinan Stomatological Hospital, Jinan, Shandong 250001, P.R. China \\ Received January 27, 2020; Accepted July 10, 2020
}

DOI: $10.3892 / 01.2021 .12549$

\begin{abstract}
Long non-coding RNAs (lncRNAs) have been confirmed to participate in cancer regulation, including oral squamous cell carcinoma (OSCC). The aim of the present study was to investigate the role of UASR1 in OSCC. The expression levels of UASR1, miR-375 and JAK2 were detected in OSCC tissues by reverse transcriptase quantitative PCR. The targets of UASR1 were predicted by IntaRNA. Colony formation and CCK-8 assays were conducted to estimate cell proliferation. Western blotting was used to detect the protein expression of JAK2. The results demonstrated that UASR1 was upregulated in OSCC tissues compared with non-tumor tissues, and the high level of UASR1 expression was associated with poor overall survival. UASR1 is predicted to interact with miR-375 and the interaction was confirmed by Dual-luciferase activity assay. However, overexpression of UASR1 and miR-375 did not affect the expression of each other. Instead, upregulation of JAK2, a target of miR-375, was observed after the overexpression of UASR1 in OSCC cells. Moreover, overexpression of UASR1 attenuated the inhibitory effects of miR-375 on the expression of JAK 2 and cell proliferation. Therefore, UASR1 is overexpressed in OSCC and regulates cancer cell proliferation by regulating the miR-375/JAK2 axis.
\end{abstract}

\section{Introduction}

As a type of malignancy developing from mouth, tongue and lips, oral cancer is one of the major subtypes of head and neck cancer (1). According to the latest GLOBOCAN

Correspondence to: Dr Lina Pan, Department of Endodontics, Shungeng Branch, Jinan Stomatological Hospital, 73 Shungeng Road, Jinan, Shandong 250001, P.R. China

E-mail:vy0017@163.com

${ }^{*}$ Contributed equally

Key words: UASR1, oral squamous cell carcinoma, miR-375, JAK2, proliferation statistics, oral cancer affected 354,864 new cases $(2.0 \%$ of all new cancer cases) and caused 177,384 deaths $(1.9 \%$ of all cancer deaths) (2). Therefore, approximately $50 \%$ of oral cancer patients succumb to this disease (3). Smoking, betel quid consumption and human papillomavirus infections are major risk factors for oral cancer (4), while the molecular pathogenesis of this disease remains largely unknown $(5,6)$, leading to difficulties in the development of anticancer treatment. In addition, the early diagnostic rate of oral cancer is low, resulting in poor survival (7).

Previous studies on the pathogenesis of oral cancer have identified a considerable number of molecular pathways involved in the development and progression of this disease $(8,9)$. In effect, some of the altered signaling pathways involved in oral cancer have been proven to be potential targets for the development of anti-cancer therapies (10). Long non-coding RNAs (1ncRNAs, $>200 \mathrm{nt}$ ) encode no proteins but participate in cancer biology by regulating cancer-related gene expression $(11,12)$. Therefore, regulating the expression of certain lncRNAs may indirectly regulate cancer-related gene expression. However, the functions of most lncRNAs in cancer remain unclear. UASR1 has been characterized as an oncogenic lnRNA in breast cancer (13); however, its roles in other types of cancer remain unknown. Our preliminary bioinformatics analysis revealed that UASR1 may interact with miR-375, which plays a tumor suppressive role by targeting JAK2 (14). The present study was therefore carried out to investigate the interactions of UASR1, miR-375 and JAK2 in oral squamous cell carcinoma (OSCC), which is a major subtype of oral cancer.

\section{Materials and methods}

Tissue collections. The present study was approved by the Ethics Committee of Jinan Stomatological Hospital (Shandong, China). A total of 62 OSCC patients (sex, 38 males and 24 females; age range, 41-69 years; mean age, $55.5 \pm 5.2$ years) who were admitted at this hospital between March 2012 and December 2014 were enrolled in this study. Diagnosis of OSCC was performed by histopathological biopsy. Patients complicated with other clinical disorders or ones with initiated therapy were excluded from this study. Patients with a 
history of malignancies were also excluded. During biopsy, paired OSCC and adjacent non-tumor tissue samples (within $5 \mathrm{~cm}$ around tumors) were collected from each patient. Fresh samples were stored in liquid nitrogen prior to use. All patients signed the informed consent.

Clinical stages and treatment. Based on AJCC staging system, the 62 patients included 14, 18, 15 and 15 cases at stage I, II, III and IV, respectively. Based on patients' clinical stages and their health conditions, treatments, such as chemotherapy, radiotherapy, surgical resections or the combination of different treatments, were performed on the patients.

Follow-up. From the day of admission, the 62 patients were followed up for 5 years to record their survival conditions. Follow-up was performed in a monthly manner through telephone. All patients completed the follow-up. Patients who succumbed to causes unrelated to OSCC were excluded.

Cell lines and culture. Normal human oral keratinocyte cells (NHOK) and OSCC cell lines (SCC25 and SCC154) were purchased from the American Type Culture Collection. NHOK cells were grown in the oral keratinocyte medium (ScienCell Research Laboratories, Inc.) and maintained at $37^{\circ} \mathrm{C}$ in a humidified chamber with $5 \% \mathrm{CO}_{2}$. The two OSCC cell lines were cultured in DMEM/F-12 (Sigma-Aldrich; Merck KGaA) supplemented with 10\% FBS (Gibco; Thermo Fisher Scientific, Inc.) and $0.4 \mu \mathrm{g} / \mathrm{ml}$ hydrocortisone and placed at $37^{\circ} \mathrm{C}$ in a humidified incubator containing $5 \% \mathrm{CO}_{2}$.

Cell transfection. SCC25 and SCC154 cells were seeded at a density of $10^{5}$ cells per well on a 6 -well plate $24 \mathrm{~h}$ prior to transfection. Once cells reached $70 \%$ confluence, they were transfected with lentivirus expressing JAK2 (shJAK2) and matched control (designated as shNC), or miR-375 mimics (designated as miR-375 mimics) and its negative control (designated as NC mimics), which were purchased from RiboBio. UASR1 expression plasmid (designated as UASR1) was obtained from Sangon Biotech, as well as the negative control (named as Vector). Lipofectamine 3000 (Invitrogen; Thermo Fisher Scientific, Inc.) was used to perform cell transfection with siRNA or miRNA mimics at a dose of $50 \mathrm{nM}$ and with vectors at a dose of $15 \mathrm{nM}$. Cells were used for subsequent experiments after $48 \mathrm{~h}$ transfection.

Dual-luciferase activity assay. Luciferase vector of UASR1 was constructed using pGL3 vector (Promega Corporation) as backbone. To perform Dual-luciferase activity assay, SCC25 and SCC154 cells were co-transfected with UASR1 expression vector + miR-375 mimic (miR-375 group) or UASR1 expression vector $+\mathrm{NC}$ miRNA (NC group). Cells were cultivated at $37^{\circ} \mathrm{C}$ for $48 \mathrm{~h}$, and the luciferase activity was analyzed using Dual-Luciferase Reporter Assay System (Promega Corporation). Renilla luciferase activity was used as the control. Each sample was assessed in triplicate.

RNA preparations. Total RNAs were extracted from SCC25 and SCC154 cells and paired tissue samples using RNAzol reagent (Sigma-Aldrich; Thermo Fisher Scientific, Inc.). RNA precipitation and washing were performed using $85 \%$ ethanol.
RNA samples were digested with gDNA eraser (Takara) to remove genomic DNAs.

$R T-q P C R$ assay. Digested RNA samples were subjected to reverse transcription using Bio-Rad iScript cDNA Kit to prepare cDNA samples (Bio-Rad Laboratories, Inc.). With cDNA samples as template, qPCR reactions were prepared using SYBR-Green Master Mix (Bio-Rad Laboratories, Inc.). The expression levels of UASR1 and JAK2 mRNA were determined with GAPDH as the endogenous control. The expression levels of mature miR-375 were detected using All-in-One ${ }^{\mathrm{TM}}$ miRNA qRT-PCR Detection Kit (GeneCopoeia) with U6 as the internal control. The sequences of the primers used were as follows: UASR1, forward, 5'-CCCTCCTCAAAC ACACATCC-3' and reverse, 5'-TTAAGGAAATTAAAAATA CC-3'; miR-375, forward, 5'-GGCTCTAGAGGGGACGAA GC-3' and reverse, 5'-GGCAAGCTTTTTCCACACCTCAGC CTTG-3'; JAK2, forward, 5'-TCTATTTTATTATGGTTT CCCTTG-3' and reverse, 5'-TTTTACTTATTTACCTCATTT CCC-3'; GAPDH, forward, 5'-GCACCGTCAAGGCTGAGA AC-3' and reverse, 5'-TGGTGAAGACGCCAGTGGA-3'; and U6, forward, 5'-GCTTCGGCAGCACATATACTAAAAT-3' and reverse, 5'-CGCTTCACGAATTTGCGTGTCAT-3'. PCR reactions were performed in triplicates and $\mathrm{Cq}$ values were processed using the $2^{-\Delta \Delta \mathrm{Cq}}$ method (15).

Western blot assay. Total proteins were isolated from cells using RIPA buffer (Invitrogen; Thermo Fisher Scientific, Inc.), followed by BCA assay (Invitrogen; Thermo Fisher Scientific, Inc.) to measure protein concentrations. Protein samples were first denatured at $95^{\circ} \mathrm{C}$ for $10 \mathrm{~min}$. Proteins $(20 \mu \mathrm{g})$ were then separated by $8-15 \%$ SDS-PAGE. Proteins were transferred to polyvinylidene fluoride (PVDF) membranes, followed by blocking in phosphate-buffered saline (PBS) containing $5 \%$ non-fat milk for $2 \mathrm{~h}$. Membranes were first incubated with rabbit primary antibodies of JAK2 (product code ab39636; Abcam) and GAPDH (product code ab9485; Abcam) at $4^{\circ} \mathrm{C}$ for $15 \mathrm{~h}$, followed by incubation at room temperature for $2 \mathrm{~h}$ with HRP IgG goat anti-rabbit (product code ab97051; Abcam). ECL solution (Sigma-Aldrich; Merck KGaA) was dropped onto membranes to develop signals. Enhanced chemiluminescence reagent (Amersham; Cytiva) was used to detect the signal on the membrane. The data were analyzed via densitometry using Image-Pro Plus software (64-bit Java 1.8.0_112; Media Cybernetics, Inc.) and normalized to expression of the internal control GAPDH.

Cell proliferation assay. Cells harvested after transfections were counted, and 4,000 cells in $0.1 \mathrm{ml}$ medium were seeded in a 96-well plate. Cells were cultured for 24, 48 or 72 h. Subsequently, $10 \mu$ l CCK-8 solution (Shanghai Yeasen Biotechnology Co., Ltd.) was added and incubated for another $2 \mathrm{~h}$ at $37^{\circ} \mathrm{C}$. The absorbance at $450 \mathrm{~nm}$ was detected using a microplate reader.

Assay of colony formation. SCC25 and SCC154 cells were seeded into 96-well plates at the density of $3 \times 10^{3}$ cells/well following transfection with shNC, pcDN3.1, NC miRNA, shJAK2, UASR1, miR-375 and UASR1 + miR-375. Two weeks later, the cells were fixed with $4 \%$ paraformaldehyde 
Table I. Associations between the expression levels of UASR1 and patients' clinical data.

\begin{tabular}{lcrrrr}
\hline Items & Cases & High & Low & $\chi^{2}$ & P-value \\
\hline Age (years) & & & & & \\
$>55$ & 30 & 14 & 16 & 0.26 & 0.61 \\
$<55$ & 32 & 17 & 15 & 1.63 & \\
Sex & & & & & \\
$\quad$ Male & 38 & 16 & 22 & 1.61 & 0.20 \\
Female & 24 & 15 & 9 & & \\
Tumor stage & & & & & \\
I & 14 & 6 & 8 & 1.17 & 0.76 \\
II & 18 & 8 & 10 & & \\
III & 15 & 9 & 6 & & \\
IV & 15 & 8 & 7 & & \\
Smoking & & & & & \\
Yes & 35 & 16 & 9 & 0.59 & 0.44 \\
No & 37 & 15 & 12 & & \\
Drinking & & & & & \\
$\quad$ Yes & 41 & 18 & 23 & 1.80 & 0.18 \\
No & 21 & 13 & 5 & & \\
\hline
\end{tabular}

(Sigma-Aldrich; Merck KGaA), stained with $0.5 \%$ crystal violet (Sigma-Aldrich; Merck KGaA) and then washed with PBS (Sigma-Aldrich; Merck KGaA). The total number of colonies from three separate transfections was counted. The average value was used to evaluate colony formation ability.

Statistical analysis. Mean \pm SD values were used to express data from 3 biological replicates. Paired t-test was used to compare differences between paired tissues. Unpaired t-test was used to compare differences between two independent groups. One-way analysis of variance (ANOVA) and Tukey's test were used to compare differences among multiple groups. The 62 OSCC patients were divided into high and low UASR 1 level groups $(n=31)$ with the median level of UASR1 expression in OSCC tissues as the cut-off value. Survival curves were plotted for the two groups based on follow-up data. Log-rank test was used to compare survival curves. Chi-squared test was performed to explore the associations between expression levels of UASR1 and patients' clinical data. $\mathrm{P}<0.05$ was statistically significant.

\section{Results}

Upregulation of UASR1 predicted poor survival of OSCC patients. The differential expression of UASR1 in OSCC was detected by measuring the expression level of UASR 1 in paired OSCC and non-tumor tissues collected from 62 OSCC patients in the present study (Table I). Compared with non-tumor tissues, expression levels of UASR1 were significantly higher in OSCC tissues (Fig. 1A, P<0.001). Survival analysis showed that patients in high UASR1 level group experienced higher mortality rate during the 5-year follow-up (Fig. 1B). It is noteworthy that treatment approaches and clinical stages were not
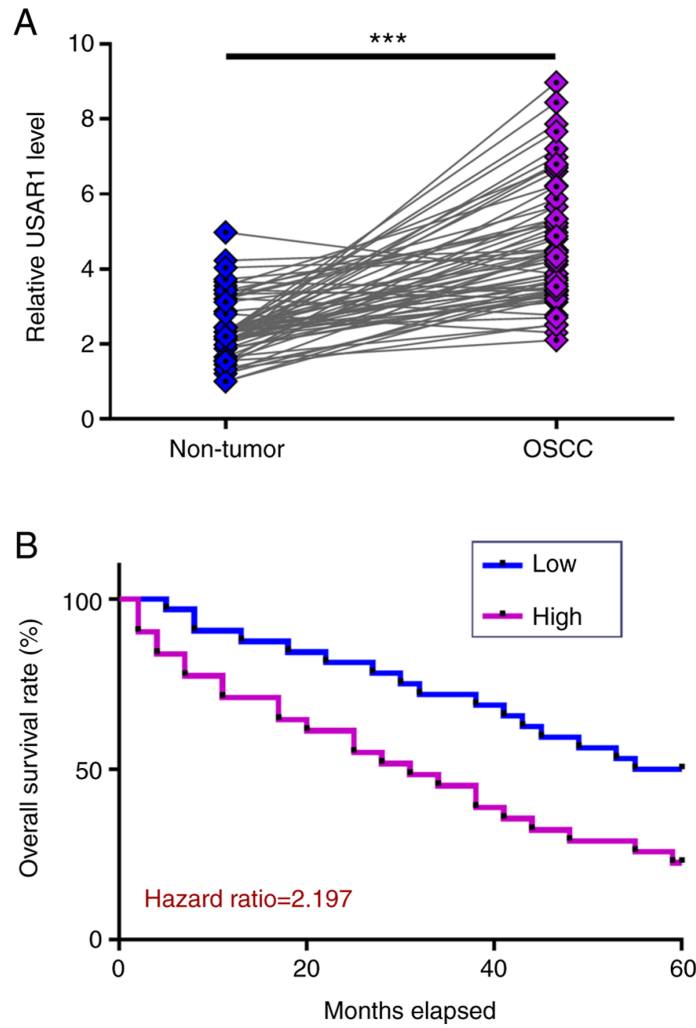

Figure 1. Upregulation of UASR1 predicted poor survival of OSCC patients. The differential expression of UASR1 in OSCC was detected by measuring the expression level of UASR1 in paired OSCC and non-tumor tissues collected from 62 OSCC patients in this study. (A) PCR reactions were repeated 3 times and mean values were presented. ${ }^{* * *} \mathrm{P}<0.001$. The 62 OSCC patients were divided into high and low UASR1 level groups $(n=31)$ with the median expression level of UASR1 in OSCC tissues as the cut-off value. Survival curves were plotted for the two groups based on follow-up data. (B) Log-rank test was used to compare survival curves.

significantly different between high and low UASR1 groups. Thus, UASR1 is likely an independent prognostic factor for OSCC. Chi-squared test showed that the expression levels of UASR1 were not significantly associated with patient age, sex, clinical stage, and smoking and drinking habits.

UASRI and miR-375 interacted with each other but did not regulate the expression of each other in SCC25 cells. IntaRNA (http://rna.informatik.uni-freiburg.de/IntaRNA/Input.jsp) was used to predict the interaction between UASR1 and miR-375. It showed that UASR 1 and miR-375 may form strong base pairs (Fig. 2A). To perform Dual-luciferase activity assay, SCC25 cells were co-transfected with UASR1 expression vector + miR-375 mimic (miR-375 group) or UASR1 expression vector $+\mathrm{NC}$ miRNA (NC group). Compared with the $\mathrm{NC}$ group, the relative luciferase activity of miR-375 group was relatively lower at $48 \mathrm{~h}$ post-transfection, indicating the direct interaction between them (Fig. 2B, $\mathrm{P}<0.05$ ). To further explore the interaction between UASR1 and miR-375, SCC25 cells were transfected with either UASR1 expression vector or miR-375 mimic, and the overexpression of UASR1 and miR-375, respectively, was confirmed by RT-qPCR (Fig. 2C, $\mathrm{P}<0.05$ ). Compared with $\mathrm{C}$ and $\mathrm{NC}$ groups, overexpression of UASR1 and miR-375 did not affect the expression of each other (Fig. 2D). 
A
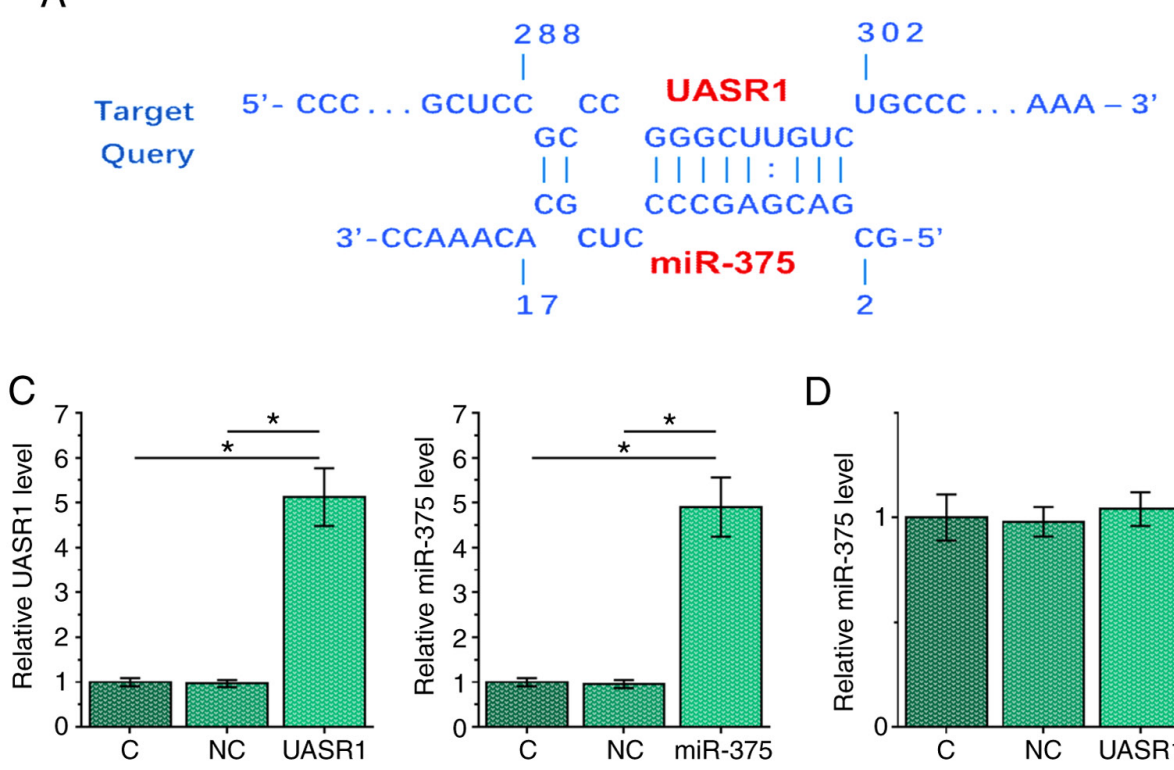

$\mathrm{D}$

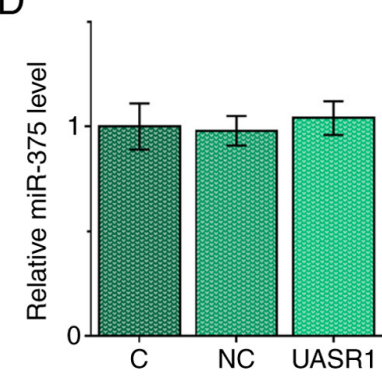

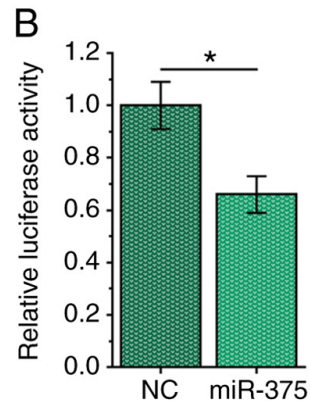

Figure 2. UASR1 and miR-375 interacted with each other but did not regulate each other. (A) lncRNA was used to predict the interaction between UASR1 and miR-375. (B) To perform Dual-luciferase activity assay, SCC25 cells were co-transfected with UASR1 expression vector + miR-375 mimic (miR-375 group) or UASR1 expression vector + NC miRNA (NC group). (C) To further explore the interaction between UASR1 and miR-375, SCC25 cells were transfected with either UASR1 expression vector or miR-375 mimic, and the overexpression of UASR1 and miR-375 was confirmed by RT-qPCR. (D) The effects of the overexpression of UASR 1 and miR-375 on each other were also analyzed by RT-qPCR. All the experiments were repeated 3 times and mean values were presented and compared; ${ }^{*} \mathrm{P}<0.05$.
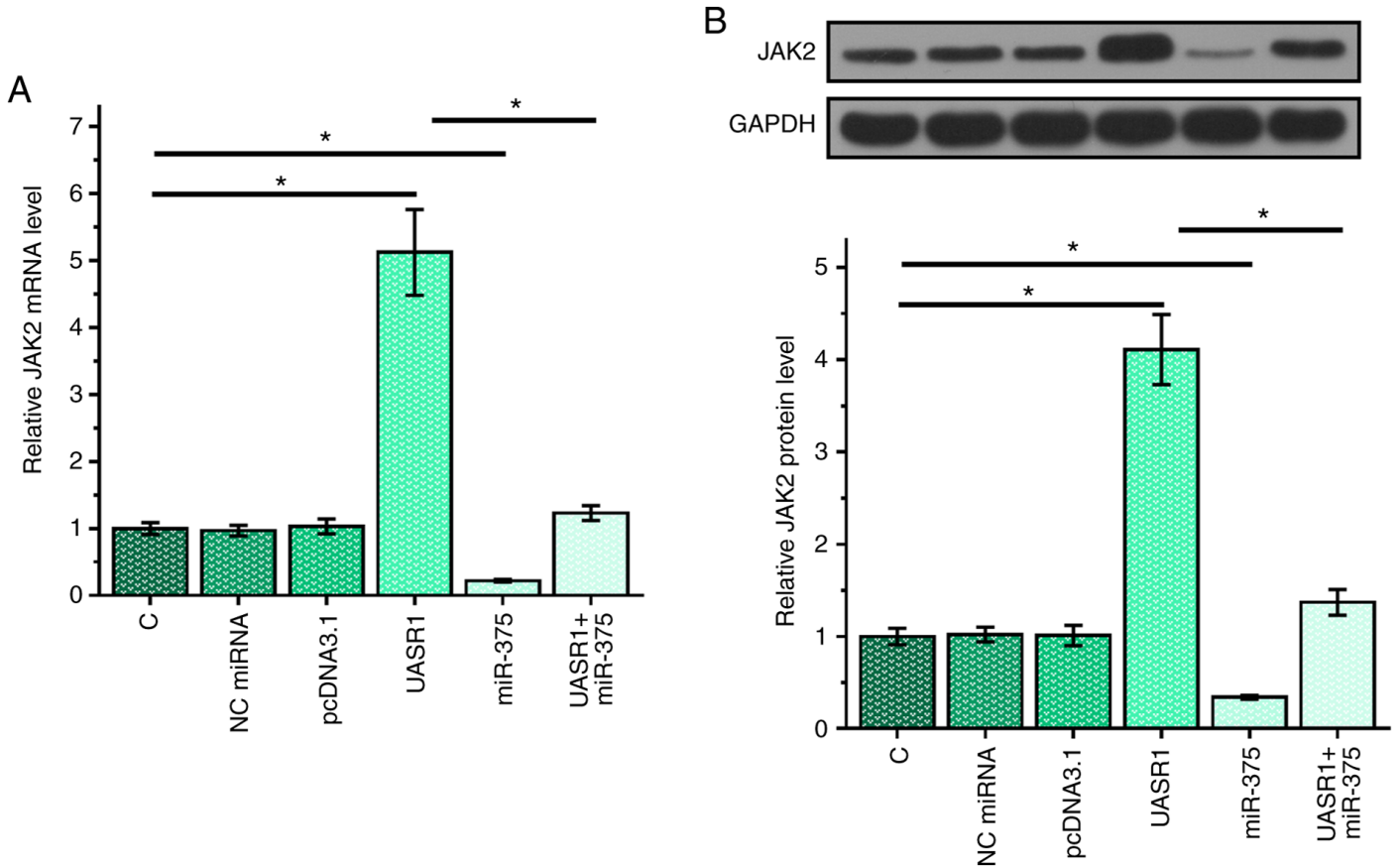

Figure 3. Overexpression of UASR1 in SCC25 cells led to upregulated JAK2. To test the possibility that UASR1 can serve as the endogenous control of miR-375, the effects of overexpression of UASR1 and miR-375 on the expression of JAK2, a target of miR-375, were assessed by (A) RT-qPCR and (B) western blot analysis. All experiments were repeated 3 times and mean values were presented and compared. ${ }^{*} \mathrm{P}<0.05$.

Overexpression of UASR1 in SCC25 cells resulted in upregulation of JAK2 in SCC25 cells. To test the possibility that UASR1 can serve as the endogenous control of miR-375, the effects of overexpressing UASR 1 and miR-375 on the expression of JAK2, a target of miR-375, were assessed by RT-qPCR (Fig. 3A) and western blots (Fig. 3B). It showed that overexpression of miR-375 led to downregulated expression of JAK2, further confirming the targeting of JAK2 by miR-375 $(\mathrm{P}<0.05)$. Overexpression of UASR 1 played an opposite role and reduced the effects of overexpressing miR-375 $(\mathrm{P}<0.0 .5)$. These data supported the role of UASR 1 as an internal control of miR-375. 
A

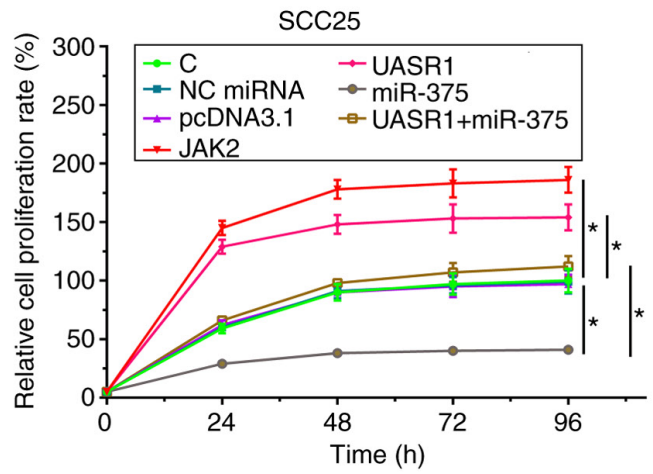

B

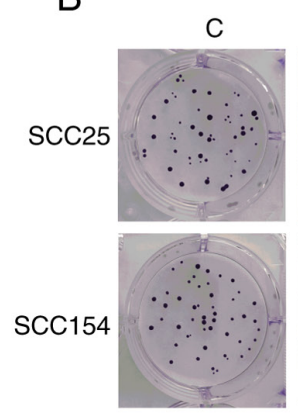

ShNC

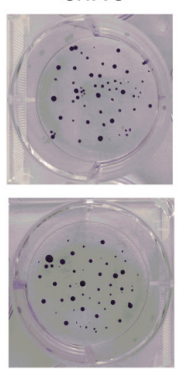

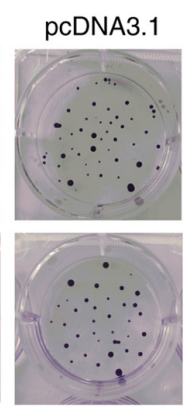

NC miRNA
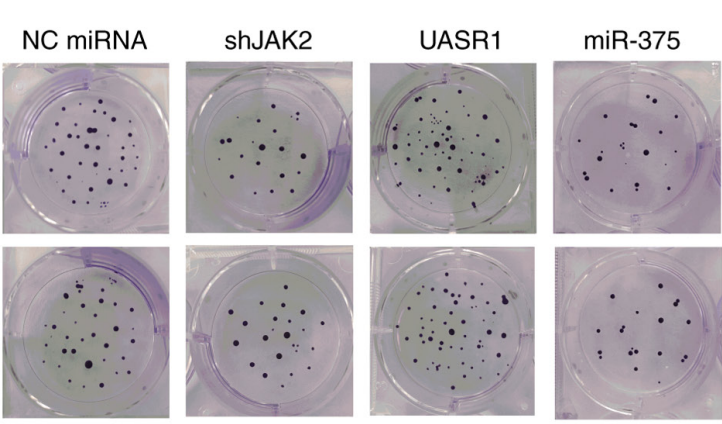

ShJAK2

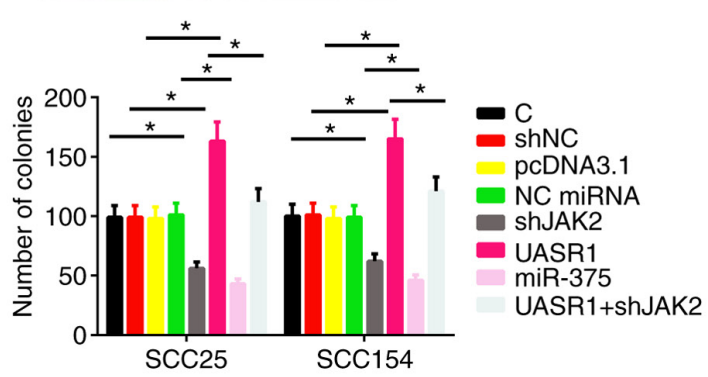

Figure 4. UASR1 promoted cell proliferation in both SCC25 and SCC154 cell lines through miR-375/JAK2 axis. (A) Effects of overexpression of UASR1, miR-375 and JAK2 on the proliferation of SCC25 cells were assessed by cell proliferation assay. (B) Effects of overexpression of UASR1, miR-375 and JAK2 on the proliferation of SCC25 cells were assessed by colony formation. All the experiments were repeated 3 times and mean values were presented and compared; ${ }^{\mathrm{P}}<0.05$.

UASR1 promoted cell proliferation in both SCC25 and SCC154 cell lines through miR-375/JAK2 axis. The effects of overexpressing UASR1, miR-375 and JAK2 on the proliferation of SCC25 and SCC154 cells were assessed by cell proliferation assay. Compared with $\mathrm{C}$ group, overexpression of UASR1 and JAK2 led to increased cell proliferation rate (Fig. 4A, $\mathrm{P}<0.05$ ). In contrast, miR-375 led to decreased cell proliferation rate $(\mathrm{P}<0.05)$. Moreover, overexpression of UASR1 reduced the effects of overexpressing miR-375 on cell proliferation $(\mathrm{P}<0.05)$. The effects of overexpressing UASR 1, miR-375 and JAK2 on the proliferation of SCC25 and SCC154 cells were assessed by colony formation. Compared with $\mathrm{C}$ group, overexpression of UASR1 led to increased cell proliferation rate. In contrast, shJAK2 and miR-375 led to decreased cell proliferation rate $(\mathrm{P}<0.05)$. Moreover, shJAK2 reduced the effects of overexpressing UASR1 on cell proliferation (Fig. 4B).

\section{Discussion}

The aim of the present study was to investigate the interactions of UASR1, miR-375 and JAK2 in OSCC. We found that UASR1 was upregulated in OSCC and it may sponge miR-375 to upregulate JAK2, thereby promoting cancer cell proliferation.

The functionality of UASR1 has only been investigated in breast cancer (13). In breast cancer, UASR1 is overexpressed and interacts with the AKT/mTOR pathway to promote the migration and proliferation of cancer cells (13). To the best of our knowledge, this study is the first to report the upregulation of UASR1 in OSCC. In addition, overexpression of UASR1 led to increased proliferation rate of OSCC cells. Therefore, UASR1 may play an oncogenic role in OSCC.

The current survival of OSCC patients with appropriate treatment is still poor $(14,16)$ and the early diagnosis of OSCC is still limited by the lack of sensitive early detection markers (13). As an alternative approach, accurate prognosis of OSCC may improve the survival of patients by guiding the determination of treatment approaches. In this study, we showed that high expression levels of UASR1 in OSCC tissues predicted poor survival of OSCC patients. However, the reliability of UASR1 as a prognostic biomarker for OSCC needs to be further confirmed by future studies with a larger sample size. 
miR-375 plays tumor suppressive roles in several types of cancer including oral cancer (17). In oral cancer, miR-375 targets SLC7A11 to suppress cancer cell proliferation (18). However, the upstream regulator of miR-375 in oral cancer remains unclear. Our study further confirmed the suppressive effects of miR-375 on the proliferation of OSCC cells. In addition, we showed that UASR 1 could serve as an internal sponge of miR-375 in OSCC cells to regulate cell behaviors. Another study reported that miR-375 could target JARK2 to suppress breast cancer cell proliferation (14). In the present study, we showed that miR-375 could also target JAK2 in OSCC to inhibit cell proliferation. Therefore, miR-375 may target multiple oncogenes in oral cancer to suppress cell proliferation. Additionally, inhibition of JAK2 significantly attenuated the effects of overexpressing UASR1 on cell proliferation, meaning that JAK2 was involved in the downstream of UASR1/miR-375 axis. However, more downstream oncogenes may serve similar roles, and need to be further investigated in future studies. Moreover, we did not find that UASR1 plays roles on the migration, invasion and apoptosis of OSCC cells, which may be further verified in other OSCC cell lines. In vivo studies should also be performed in future to verify the findings of the present study. In conclusion, UASR1 is upregulated in OSCC and predicts poor survival. UASR1 may sponge miR-375 to upregulate JAK2, thereby promoting cancer cell proliferation.

\section{Acknowledgements}

Not applicable.

\section{Funding}

Not applicable.

\section{Availability of data and materials}

The datasets used and/or analyzed during the current study are available from the corresponding author on reasonable request.

\section{Authors' contributions}

YS designed the study. YS, LC and LP organized the data, performed the data analyses and produced the initial draft of the manuscript. YS, LC and LP performed the experiments and generated the figures. All authors have read and approved the final version.

\section{Ethics approval and consent to participate}

The present study was approved by the Ethics Committee of Jinan Stomatological Hospital (Shandong, China). All patients signed informed consent.

\section{Patient consent for publication}

Not applicable.

\section{Competing interests}

The authors declare that they have no competing interests.

\section{References}

1. Lydiatt WM, Patel SG, O'Sullivan B, Brandwein MS, Ridge JA, Migliacci JC, Loomis AM and Shah JP: Head and neck cancers-major changes in the American joint committee on cancer eighth edition cancer staging manual. CA Cancer J Clin 67: 122-137, 2017.

2. Bray F, Ferlay J, Soerjomataram I, Siegel RL, Torre LA and Jemal A: Global cancer statistics 2018: GLOBOCAN estimates of incidence and mortality worldwide for 36 cancers in 185 countries. CA Cancer J Clin 68: 394-424, 2018.

3. Kumar M, Nanavati R, Modi TG and Dobariya C: Oral cancer: Etiology and risk factors: A review. J Cancer Res Ther 12: 458-463, 2016

4. Ghantous Y and Abu Elnaaj I: Global incidence and risk factors of oral cancer. Harefuah 156: 645-649, 2017 (In Hebrew).

5. Rivera C: Essentials of oral cancer. Int J Clin Exp Pathol 8: 11884-11894, 2015.

6. Ram H, Sarkar J, Kumar H, Konwar R, Bhatt ML and Mohammad S: Oral cancer: Risk factors and molecular pathogenesis. J Maxillofac Oral Surg 10: 132-137, 2011.

7. Brocklehurst P, Kujan O, O'Malley LA, Ogden G, Shepherd S and Glenny AM: Screening programmes for the early detection and prevention of oral cancer. Cochrane Database Syst Rev: CD004150, 2013.

8. Mishra R: Glycogen synthase kinase 3 beta: Can it be a target for oral cancer. Mol Cancer 9: 144, 2010.

9. Molinolo AA, Marsh C, El Dinali M, Gangane N, Jennison K, Hewitt S, Patel V, Seiwert TY and Gutkind JS: mTOR as a molecular target in HPV-associated oral and cervical squamous carcinomas. Clin Cancer Res 18: 2558-2568, 2012.

10. Ohnishi Y, Yasui $H$, Nozaki $M$ and Nakajima $M$ : Molecularly-targeted therapy for the oral cancer stem cells. Jpn Dent Sci Rev 54: 88-103, 2018.

11. Sanchez Calle A, Kawamura Y, Yamamoto Y, Takeshita F and Ochiya T: Emerging roles of long non-coding RNA in cancer. Cancer Sci 109: 2093-2100, 2018.

12. Gutschner T and Diederichs S: The hallmarks of cancer: A long non-coding RNA point of view. RNA Biol 9: 703-719, 2012

13. Cao Z, Wu P, Su M, Ling H, Khoshaba R, Huang C, Gao H, Zhao Y, Chen J, Liao Q, et al: Long non-coding RNA UASR1 promotes proliferation and migration of breast cancer cells through the AKT/mTOR pathway. J Cancer 10: 2025-2034, 2019.

14. Ding L, Xu Y, Zhang W, Deng Y, Si M, Du Y, Yao H, Liu X, Ke Y, Si J and Zhou T: MiR-375 frequently downregulated in gastric cancer inhibits cell proliferation by targeting JAK2. Cell Res 20: 784-793, 2010.

15. Livak KJ and Schmittgen TD: Analysis of relative gene expression data using real-time quantitative PCR and the 2(-Delta Delta C(T)) method. Methods 25: 402-408, 2001.

16. Tirelli G, Gatto A, Boscolo Nata F, Bussani R, Piccinato A, Marcuzzo AV and Tofanelli M: Prognosis of oral cancer: A comparison of the staging systems given in the 7th and 8th editions of the American Joint Committee on Cancer Staging Manual. Br J Oral Maxillofac Surg 56: 8-13, 2018.

17. Jairajpuri ZS, Rana S, Hajela A and Jetley S: Toward early diagnosis of oral cancer: Diagnostic utility of cytomorphological features, a pilot study. Natl J Maxillofac Surg 10: 20-26, 2019.

18. Wu Y, Sun X, Song B, Qiu X and Zhao J: MiR-375/SLC7A11 axis regulates oral squamous cell carcinoma proliferation and invasion. Cancer Med 6: 1686-1697, 2017. International (CC BY-NC-ND 4.0) License. 